ARTICLE

Corrected: Publisher correction

DOI: $10.1038 /$ s41467-018-03498-0 OPEN

\title{
RING tetramerization is required for nuclear body biogenesis and PML sumoylation
}

\author{
Pengran Wang ${ }^{1,2}$, Shirine Benhenda ${ }^{3,4}$, Haiyan Wu ${ }^{1,5}$, Valérie Lallemand-Breitenbach ${ }^{3,4,6}$, Tao Zhen ${ }^{1}$ \\ Florence Jollivet ${ }^{3,4}$, Laurent Peres ${ }^{3,4}$, Yuwen Li ${ }^{1}$, Sai-Juan Chen ${ }^{1,2,4,5}$, Zhu Chen 1,2,4,5, Hugues de Thé ${ }^{3,4,6,7}$ \\ \& Guoyu Meng (1D 1,4
}

ProMyelocyticLeukemia nuclear bodies (PML NBs) are stress-regulated domains directly implicated in acute promyelocytic leukemia eradication. Most TRIM family members bind ubiquitin E2s and many acquire ligase activity upon RING dimerization. In contrast, PML binds UBC9, the SUMO E2 enzyme. Here, using X-ray crystallography and SAXS characterization, we demonstrate that PML RING tetramerizes through highly conserved PML-specific sequences, which are required for NB assembly and PML sumoylation. Conserved residues implicated in RING dimerization of other TRIMs also contribute to PML tetramer stability. Wild-type PML rescues the ability of some RING mutants to form NBs as well as their sumoylation. Impaired RING tetramerization abolishes PML/RARA-driven leukemogenesis in vivo and arsenic-induced differentiation ex vivo. Our studies thus identify RING tetramerization as a key step in the NB macro-molecular scaffolding. They suggest that higher order RING interactions allow efficient UBC9 recruitment and thus change the biochemical nature of TRIM-facilitated post-translational modifications.

\footnotetext{
${ }^{1}$ State Key Laboratory of Medical Genomics, Shanghai Institute of Hematology, Rui Jin Hospital affiliated to Shanghai Jiao Tong University School of Medicine, 197 Ruijin Er Road, Shanghai 200025, China. ${ }^{2}$ Institute of Health Sciences, Shanghai Institutes for Biological Sciences and Graduate School, Chinese Academy of Sciences, 320 Yueyang Road, Shanghai 200031, China. ${ }^{3}$ University Paris Diderot, Sorbonne Paris Cité, INSERM U944, CNRS UMR7212, Equipe labellisée LNCC, Hôpital St. Louis 1, Paris 75475, France. ${ }^{4}$ Laboratoire International Associé, Hematology and Cancer, RuiJin Hospital, INSERM and CNRS, Shanghai, China. ${ }^{5}$ Key Laboratory of Systems Biomedicine, Shanghai Center for Systems Biomedicine, Shanghai Jiao Tong University, 800 Dong Chuan Road, Shanghai 200240, China. ${ }^{6}$ Collège de France, Paris Sciences Lettres research university, 11 place Marcelin Berthelot, 75005 Paris, France. 7 Service de Biochimie, Hôpital St. Louis, Assistance Publique Hôpitaux de Paris, Paris 75475, France. These authors contributed equally: Pengran Wang, Shirine Benhenda, Haiyan Wu, Valérie Lallemand-Breitenbach. These authors jointly supervised this work: Guoyu Meng, Hugues de Thé. Correspondence and requests for materials should be addressed to Z.C. (email: zchen@stn.sh.cn) or to H.Thé. (email: hugues.dethe@inserm.fr) or to G.M. (email: guoyumeng@shsmu.edu.cn)
} 
$\mathrm{P}$ ML nuclear bodies (NBs) are membrane-less insoluble structures whose assembly increases upon stress and which recruit a large number of partner proteins ${ }^{1}$. PML NBs also recruit enzymes implicated in several post-translational modifications, primarily UBC9, the key E2 SUMO-conjugating enzyme, possibly facilitating partner sumoylation ${ }^{2,3}$. There is evidence that PML NBs regulate stress responses, in particular senescence induction, at least in part through the control of p53 activation $^{4-6}$. Indeed, NBs are directly implicated in the eradication of acute promyelocytic leukemia (APL) by retinoic acid and arsenic therapy, notably by inducing p53-mediated senescence $^{7-9}$. PML belongs to the TRIM family of proteins, defined by the presence of a RING domain, one or two other zinc fingers (B boxes) and a long coiled coil ${ }^{10}$. Many TRIMs are ubiquitin E3 ligases, through their ability to bridge RING-binding E2 enzymes to specific substrates ${ }^{11}$. Furthermore, TRIM RING dimerization is often required for E2 interaction ${ }^{12-14}$. Interestingly, PML RING interacts with UBC9 ${ }^{15}$. Similar to PML, several TRIM family members are interferon-induced, regulate innate immunity and/ or assemble into large nuclear or cytoplasmic complexes ${ }^{12,14}$. PML RING, B boxes and coiled coil are all important for NBbiogenesis $^{16-18}$. Although PML sumoylation was proposed to drive PML NB-biogenesis ${ }^{19,20}$, SUMO is required for NB client protein recruitment rather than PML selfassembly into $\mathrm{NBs}^{2,21-23}$.

Here we report the crystallographic high-resolution structure of PML RING, and demonstrate that it assembles into a tetrameric torus. Interfaces of these tetramers involve PML-specific sequences that are highly conserved during evolution. Critically, their mutation prevents PML tetramerization in solution and abolishes NB formation and PML sumoylation in cellulo. Our data suggest that this novel macro-molecular RING assembly may control interactions with E2s and hence TRIM functions.

\section{Results}

RING crystal structure reveals a torus-shaped tetramer. We have determined the crystal structure of PML RING at a $1.6 \AA$ resolution by single wavelength anomalous dispersion (Fig. 1a, Supplementary Figure 1a-e). Similar to previous RING structures ${ }^{16,24}$, the folding of PML RING $_{49-104}$ is coordinated by two Zn ions, but in contrast with previous studies, PML RING leads tetrameric complexes. Within a tetramer, each PML subunit adopts a balloon shape configuration with two distinct subdomains. Sub-domain $1\left(\mathrm{SD}_{\mathrm{FQF}}\right)$ is a loop containing only three residues, $\mathrm{F}_{52} \mathrm{Q}_{53} \mathrm{~F}_{54}$, while sub-domain 2 (SD2), comprising residues 55-99, is a classic $\mathrm{C}_{3} \mathrm{HC}_{4}$ RING finger (Fig. 1a,b). Together with homo-dimerization of $\mathrm{SD} 2$, interactions between $\mathrm{SD} 1_{\mathrm{FQF}}$ and SD2 create a four subunits torus-like structure (Fig. 1). Importantly, the residues involved in both interfaces are not only highly conserved among PML orthologs (purple boxes in Fig. 1b), but also highly specific to PML within the TRIM family. The RING tetramer harbors four highly charged patches at each side of the torus as well as a symmetrical deep groove diagonally across each face (Supplementary Figure 1a-e), which could constitute binding sites for partner proteins and/or other PML domains (see below). The interactions between $\mathrm{SD} 1_{\mathrm{FQF}} / \mathrm{SD} 2$ and SD2/SD2 interfaces of the PML RING tetramer are mediated by: (i) the interaction between $\mathrm{F} 52$ from $\mathrm{SD}_{\mathrm{FQF}}$ loop of subunit 1 , and the hydrophobic pocket delineated by the side chains of L70, L81, W95 from SD2 of subunit 2 (Fig. 1c); (ii) the hydrophobic interactions between $\mathrm{F} 54$ from $\mathrm{SD} 1_{\mathrm{FQF}}$, K65 from SD2 of the same subunit 1 and K68 from SD2 of subunit 2, so that the benzyl side chain of F54 is sandwiched by the side chains of K65 and K68 (Fig.1c); (iii) an intermolecular disulfide bridge C66-C66 in the crystal; (iv) two adjacent SD2 subunits pack against each other in a face-to-face configuration mainly mediated by the L73L73 hand-shake-like hydrophobic interaction, but also by a highly evolutionarily conserved loop around C91 (Fig. 1a,b,d).

When the previous NMR RING structure is superimposed with the crystallographic one, significant differences appear (Fig. 1e). In the previously described monomeric structure, $\mathrm{SD} 1_{\mathrm{FQF}}$ was proposed to interact with the SD2 of the same subunit. Within the current tetramer, the $\mathrm{N}$-terminal $\mathrm{F}_{52} \mathrm{Q}_{53} \mathrm{~F}_{54} \mathrm{SD} 1_{\mathrm{FQF}}$ undergoes a radical $23 \AA$ swing away from the adjacent SD2, allowing the $\mathrm{SD} 1_{\mathrm{FQF}}$ loop to engage with the hydrophobic pocket of the other subunit. This shift also exposes L73 and allows SD2 dimerization (Fig. 1e,f). As estimated by the AREAIMOL ${ }^{25}$ program, the buried surfaces between one set of $\mathrm{SD}_{\mathrm{FQF}} / \mathrm{SD} 2$ and SD2/SD2 dimers are 1333 and $779 \AA^{2}$, respectively (Fig. 1f). As oligomerization proceeds, the buried surface of PML RING tetramer increases significantly to $4216 \AA^{2}$, accounting for $\sim 40 \%$ of the overall surface of PML RING tetramer (Fig. 1f).

Tetramerizationin solution through highly conserved sequences. Existence of PML RING tetramers is supported by biochemical analyses: analytical ultracentrifuge experiments of PML $\mathrm{RING}_{49-104}$ showed three peaks corresponding to molecular masses of 7.5, 15 and $34 \mathrm{kDa}$, consistent with RING monomer, dimer and tetramer formation (Fig. 2a). Importantly, disruption of either interfaces abolished complex formation (Fig. 2b), while not altering the RING overall fold (Supplementary Figure 2). In order to further document PML RING tetramerization in solution, we used small-angle-X-ray scattering (SAXS) (Fig. 2c). The match in crystal fitting with a $X^{2}$ value of 1.19 suggested the existence of PML RING monomer (60.1\%), dimer $(26.1 \%)$ and tetramer $(13.8 \%)$ in solution (Fig. $2 \mathrm{~d}$ ). We then performed gel filtration of PML deleted for B boxes and coiled coil domains $(\triangle \mathrm{BC})$ to prevent oligomerization, with or without F52/54E or L73E, or with mutations of the $\mathrm{SD} 1_{\mathrm{FOF}^{-}}$-interacting amino acid K65, K68 and L81 in SD2 (Supplementary Figure 4a). These PML mutants were consistently found in lower molecular weight fractions compared to PML $\triangle B C$, suggesting that they lost their self-interaction properties. Similarly, when the F52/54E mutant was mixed with PML $\triangle B C$, the latter was found in lower molecular weight fractions, suggestive for impairment of its tetramerization (Supplementary Figure 4b).

Tetramers are also stabilized by TRIM-conserved sequence. Other TRIM RING domains form dimers rather than tetramers, prompting a detailed analysis of the evolutionary diversity between PML- and TRIM- RING sequences (Fig. 3a and Supplementary Figure 3). Among TRIM RINGs, interactions between $\mathrm{N}$-terminal and C-terminal helix segments were recently implicated in TRIM5, TRIM25 or TRIM32 dimer formation ${ }^{26-29}$. While the N-terminal sequence is not found in PML RING, the highly conserved C-terminal amino acids are present in PML, with N106 and L122 being the two most conserved residues in this TRIM-specific helix (Fig. 3a). An extended PML sequence, RING $_{1-119}$ was thus subjected to gel filtration and SEC-MALS analysis. Critically, we obtained evidence for stable tetrameric and dimeric assembly in solution (Fig. 3b-e). Mutations of either of N106R or L112R inhibited PML RING tetramerization (Fig. 3f). Critically, mutations of residues implicated in the tetramer interfaces $\left(\mathrm{F}_{52} \mathrm{QF}_{54}\right.$ and $\left.\mathrm{L} 73\right)$, which do not alter the RING overall fold (Supplementary Figure 2), also precluded tetramerization of the extended RING (Fig. 3f and Supplementary Figure 4). We finally subjected the long version of PML RING to limited proteolysis and mass sequencing, revealing that residues 53-98 constitute its structured core domain (Fig. $3 \mathrm{~g}$ and Supplementary Figure 5). Remarkably, this domain is almost identical to the one 
a
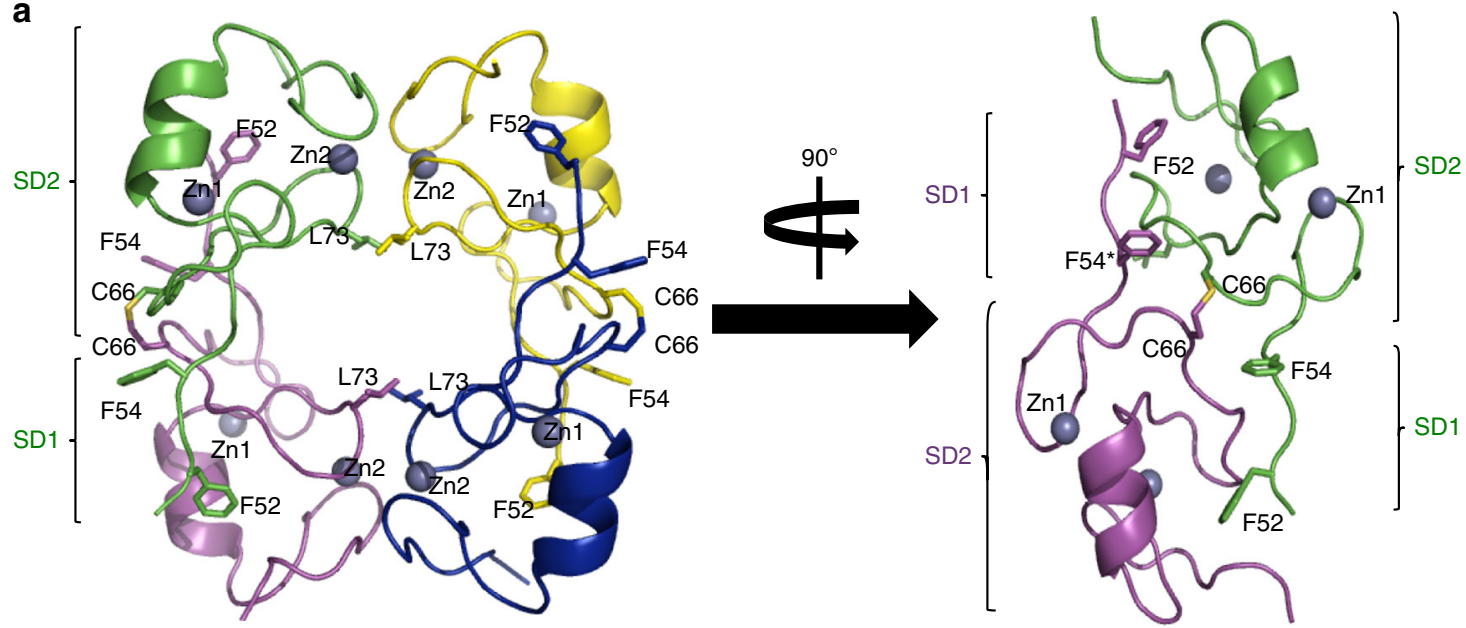

b

\begin{tabular}{|c|c|}
\hline & 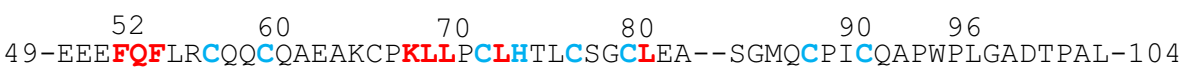 \\
\hline Macaca & EEEFQFLRCQQCQAEAKCPKLLPCLHTLCSGCLEE--SGMQCPICQAPWPLGADTPAL \\
\hline Mouse & QEEFQFLRCPSCQAQAKCPKLLPCLHTLCSGCLEA--PGLQCPICKAPGQADANGEAL \\
\hline Bat & EEEFQFLRCHGCQAEAKCPKLLLCLHTLCSGCLQA--PDMQCP ICQASLPPGADGPVL \\
\hline Chicken & EGDFQFVLCEGCRQESPNLKLLTCLHTLCLGCLRENKPVGQCPVCQAPI PQPDGIPNV \\
\hline rtle & EEEFOFLLCQGCQKE PRNPKLLSCLHTLCTDCLEENKPVGQCPICQAPI PQASGIPDQ \\
\hline
\end{tabular}

C
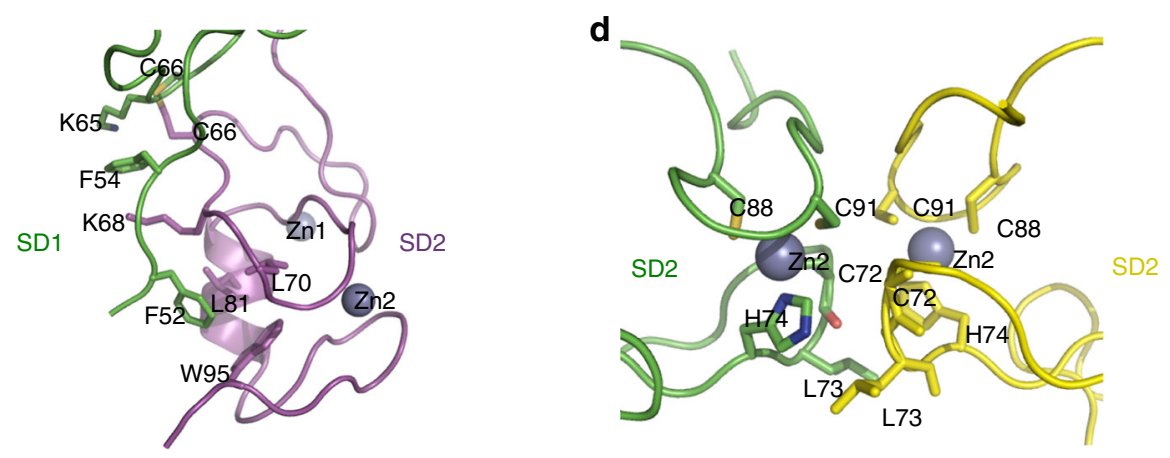

e
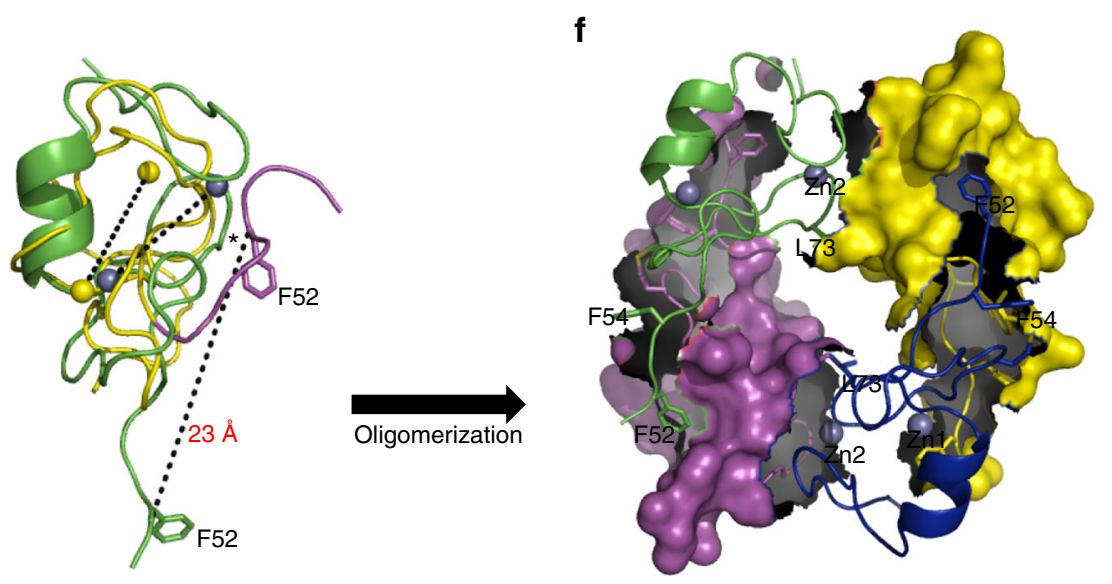

Fig. 1 Crystal structure of PML RING tetramer. a Crystal structure of PML RING tetramer. The residues 51-97 of crystallized PML RING49-104 are visible in the electron density map. Four PML monomers are colored in green, magenta, blue and yellow, respectively. The contact residues (F52, F54 and L73) are shown in stick representation. $\mathrm{Zn}$ ions are shown in sphere representation. Sub-domain 1 (SD1 $1_{\mathrm{FQF}}$ ) and sub-domain 2 (SD2) are bracketed. b Sequence alignment of the PML RING domains from different species. The conserved residues lying in the F52/54-interfaces and L73-interfaces are highlighted in red, while the conserved $\mathrm{Zn}$-binding residues are colored in cyan. The deep purple boxes underneath the sequences are used to highlight the conserved (greater than 5 out of 6 ) residues among PML RING. c, d Enlarged views of PML RING dimeric interfaces. The residues involving PML oligomerization are shown in stick representation. e Structural superimposition between different PML RINGs. The NMR ${ }^{16}$ and crystallographic PML RINGs are colored in yellow and green, respectively. $L 73$ positions are labeled with "Asterisk". The internal $\mathrm{Zn}-\mathrm{Zn}$ distances and the putative $\mathrm{F}_{52} \mathrm{Q}_{53} \mathrm{~F}_{54}$ swing are highlighted with dash lines. $\mathbf{f}$ The buried areas of the SD1 $1_{\mathrm{FQF}} / \mathrm{SD} 2$ and SD2/SD2 interfaces are shown in gray 

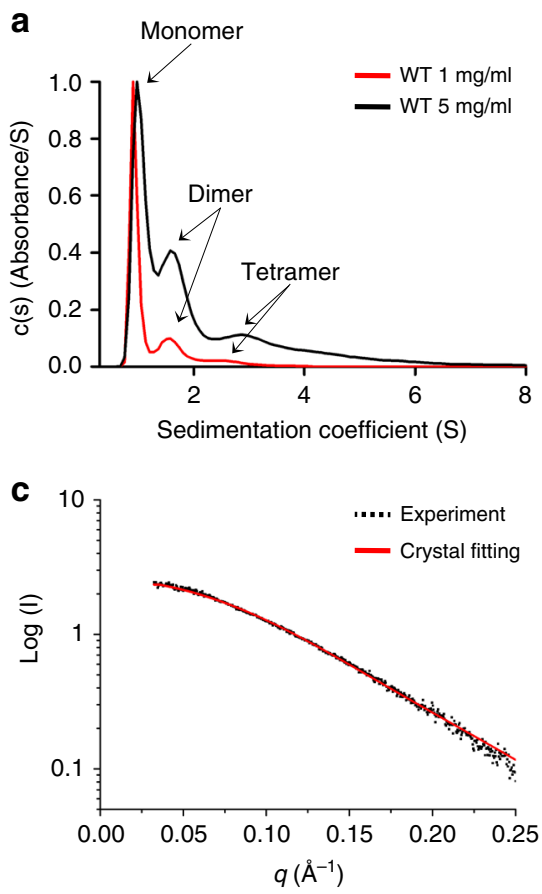

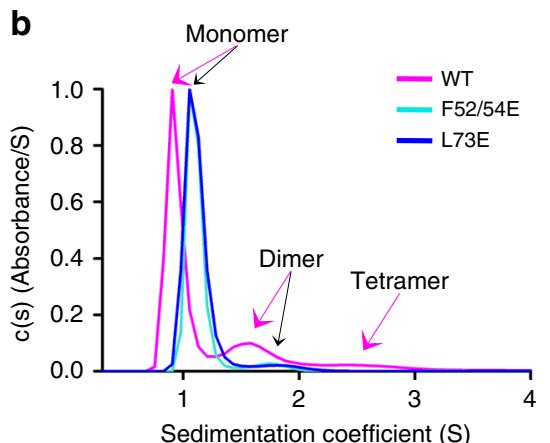

d

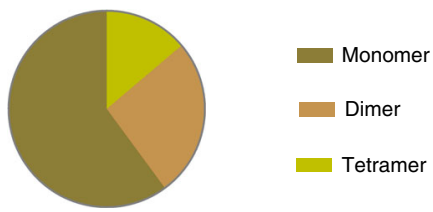

Fig. 2 Characterization of PML RING tetramers. a Analytical ultracentrifugation analysis of tetramerization of wild type PML RING, at 1 and 5 mg ml ${ }^{-1}$ concentrations, respectively. b Analytical ultracentrifugation analysis of $1 \mathrm{mg} \mathrm{ml}^{-1}$ PML RING, PML RING F52/54E and PML RINGL73E. c SAXS characterization. Black dots, experimental data. Red line, the theoretical scattering pattern derived from mixs of PML RING multimers. d Oligomeric distribution estimated by SAXS analysis. The distribution of PML RING monomer (60.1\%), dimer (26.1\%) and tetramer (13.8\%) was estimated using the OLIGOMER algorithm

used for PML RING crystallization, suggesting that the Cterminal helix bundle may somehow be dissociated from the RING core. Collectively, these biochemical studies support the importance of the RING tetramer interfaces for higher order PML RING interactions.

NB assembly and PML sumoylation requires RING tetramerization. To test whether PML RING tetramerization is also important in cellulo, we explored NBs formation and PML sumoylation by stable expression of the mutants in immortalized $\mathrm{Pml}^{-1}-$ fibroblasts (Fig. 4). Critically, these mutants generated significantly fewer $\mathrm{NBs}\left(\mathrm{PML}_{\mathrm{F} 52 / 54 \mathrm{E}}\right)$ or even none $\left(\mathrm{PML}_{\mathrm{L} 73 \mathrm{E}}\right)$, while the diffuse nuclear staining was dramatically increased. The $\mathrm{PML}_{\mathrm{F} 52 / 54 \mathrm{~A}}$ mutant behaved as $\mathrm{PML}_{\mathrm{F} 52 / 54 \mathrm{E}}$. The effects of single $\mathrm{F}$ to $\mathrm{E}$ mutations were less drastic, but nevertheless significantly decreased the numbers of NBs (Supplementary Figure 6a). Similarly, combining mutations of the residues facing F52 and F54 on SD2 (K65/68A-L81E and K65/68A-W95E) also reduced NB formation and increased the nuclear diffuse fraction of PML (Supplementary Figure 6d). In contrast, mutations of C66, which is not evolutionarily conserved (Fig.1b), to S or A had modest or no effect on NB biogenesis or basal sumoylation (Supplementary Figure $6 \mathrm{~d}, \mathrm{~g}$ ). Critically, $\mathrm{PML}_{\mathrm{F} 52 / 54 \mathrm{E}}$ or $\mathrm{PML}_{\mathrm{L} 73 \mathrm{E}}$ failed to undergo efficient sumoylation (Fig. 4b). The more subtle mutants of the $\mathrm{SD} 1_{\mathrm{FQF}} / \mathrm{SD} 2$ interface showed decreased sumoylation (Supplementary Figure $6 \mathrm{~b}, \mathrm{c}, \mathrm{e}, \mathrm{f})$. Mutations in the RING C-terminal conserved TRIM dimeric interface (N106Rand/or L112R, as well as N106A/L112A) also impaired NB biogenesis and sumoylation (Supplementary Figure 6h,i). Collectively, these observations argue that PML RING tetramerization is essential for $\mathrm{NB}$ assembly and subsequent PML sumoylation in cellulo.

Trans-complementation assays revealed that co-expression of CFP-WT PML fully restored NBs assembly of $\mathrm{HA}-\mathrm{PML}_{\mathrm{F} 52 / 54 \mathrm{E}}$, but not that of HA-PML $\mathrm{L}_{\mathrm{L} 3 \mathrm{E}}$ which remained diffusely distributed despite a few aggregates (Fig. 5a and Supplementary Figure 7a). This difference in NB re-assembly suggests that the two L73-L73 interactions are required to stabilize the tetrameric torus, while fewer than four $\mathrm{SD} 1_{\mathrm{FQF}} / \mathrm{SD} 2$ interfaces may be sufficient. PML RING interacts with UBC9 and PML NBs efficiently recruit UBC9, especially under arsenic-induced oxidative stress ${ }^{2,15}$ (Fig. 5c). Critically, in our trans-complementation assays, CFPWT PML co-expression restores efficient basal or arsenicenhanced sumoylation of $\mathrm{HA}-\mathrm{PML}_{\mathrm{F} 52 / 54 \mathrm{E}}$, but never that of $\mathrm{PML}_{\mathrm{L} 73 \mathrm{E}}$, mirroring NB-reformation (Fig. 5b and Supplementary Figure $7 \mathrm{~b}$ ). We then performed mammalian two-hybrid experiments with either full-length PML or its RING domain. Both demonstrated interaction of PML RING with UBC9, but not RING tetramer mutants (Fig. 5d and Supplementary Figure 7e). The rare NBs formed by $\mathrm{PML}_{\mathrm{F} 52 / 54 \mathrm{~A}}$ did colocalize with UBC9, but FRAP experiment demonstrated a decreased time of halfrecovery, suggesting thatdecreased affinity of this PML mutant with UBC9 may explain its inefficient SUMO-conjugation (Supplementary Figure 7c,d). Collectively, these observations support a role of RING tetramers for NB formation, direct or indirect UBC9 recruitment and PML sumoylation.

APL development and arsenic response require tetramerization. Using PML/RARA transgenic mice, we investigated whether PML RING tetramerization is important in APL development. PML/RARA, but not PML/RARA ${ }_{\text {L73E }}$, efficiently developed APL (Fig. 6a), in line with our observation that sumoylation of PML moiety is required for efficient leukemogenesis in vivo ${ }^{30}$. Previous studies have shown that the therapeutic effect of arsenic in APL is based on its ability to trigger PML or PML/RARA re-assembly in NBs, hyper-sumoylation and subsequent PML/RARA degradation $^{7,21,31-33}$. Arsenic was unable to trigger NBs re-assembly and 

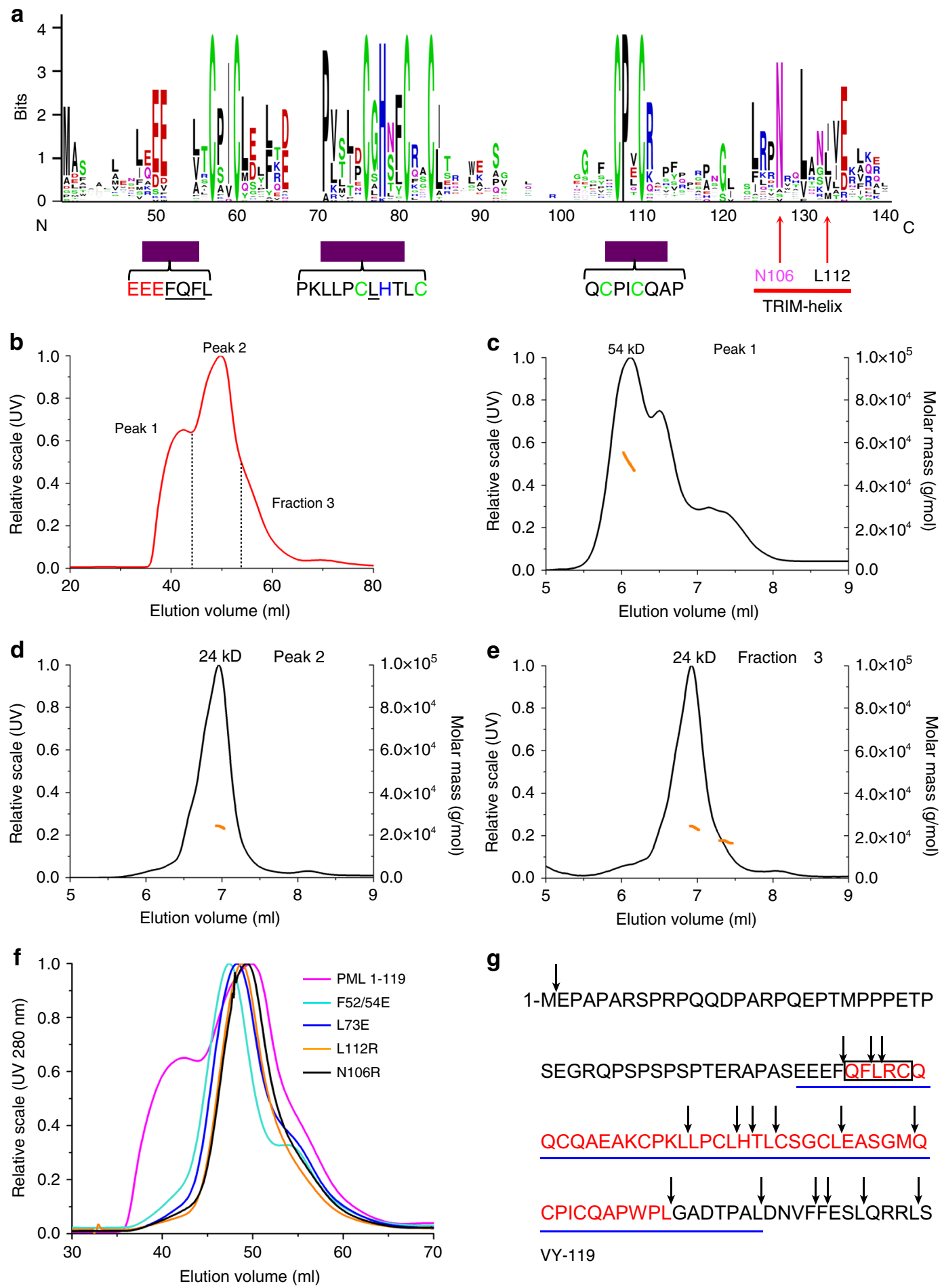

g
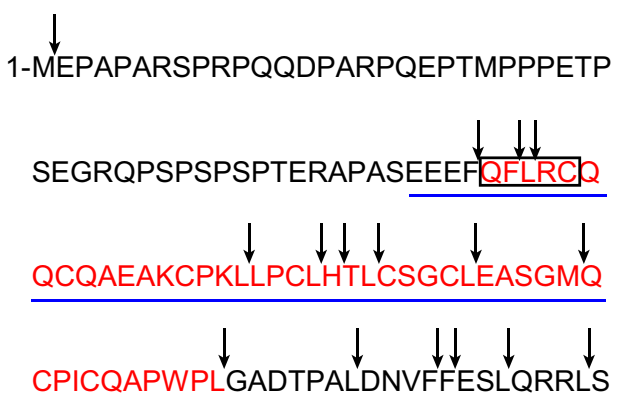

VY-119

Fig. 3 Biochemical evidence for RING tetramer formation. a Consensus sequence among TRIM RINGs (Top). PML conserved sequences are highlighted with purple boxes (Bottom). The conserved TRIM dimeric interface is highlighted with a red line. The Asn and lle/Leu residues (red arrows) mediate dimeric assembly of other TRIMs. b Gel filtration analysis of recombinant PML RING 1 -119. Two elution peaks and a last fraction, designated as Peak 1,2 and fraction 3, respectively. c-e SEC-MALS reanalysis of Peak 1 (c), Peak 2 (d) and fraction 3 (e) derived from previous gel filtration (b). Black curve, the elution profile in UV. Orange curve, the estimated molecular weight. The theoretical molecular weight of PML RING $1-119$ is $13.1 \mathrm{kDa}$. Peak 1 sample displayed distinct peaks that correspond to the tetramer $(54 \mathrm{kDa})$, dimer and monomer in the solution. Peak 2 and fraction 3 samples primarily exhibit RING dimer (24 kDa). f Gel filtration of WT RING $1-119$ and its mutants. $\mathbf{g}$ Recombinant PML RING $1-119$ was subjected to chymotrypsin digestion (Supplementary Figure 5). Arrows indicate the chymotrypsin sites and amino acids indicated in red correspond to the protected fragment, as determined by mass spectrometry and $\mathrm{N}$ terminal sequencing (Box). RING $49-104$ sequences analyzed by $X$-ray crystallography are underlined with a blue line 
a

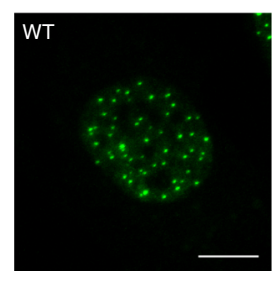

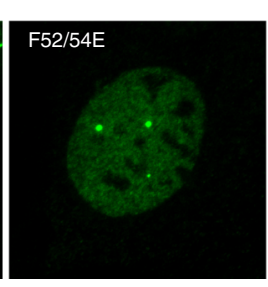

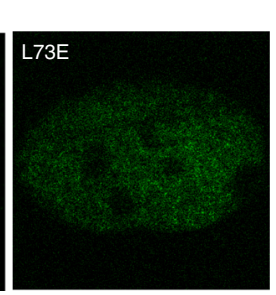

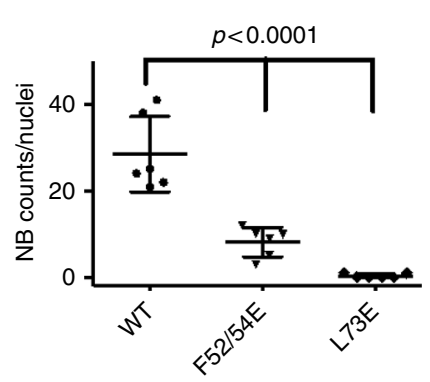

b

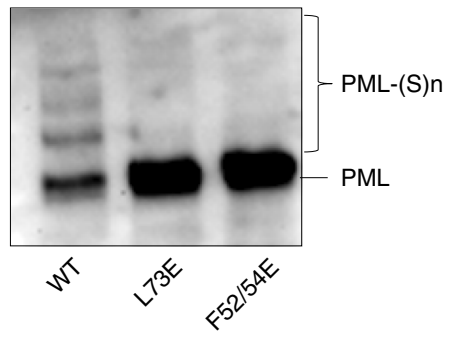

Fig. 4 PML tetramerization in NB assembly and PML sumoylation in cellulo. a Pml-/- MEFs stably expressing wild type HA-PML, HA-PMLF52/54E and HAPML L73E were analyzed by immuno-fluorescence using anti-HA antibodies (left, green). Scale bar is $5 \mu \mathrm{m}$. Statistical analyses (right). All experiments have been done in five independent replicates, NB count was from 10 to 20 nuclei. Values are means \pm S.D. and One-way ANOVA ( $p$-values) are indicated. b Western blot analysis demonstrating loss of sumoylation of these PML mutants, as detected by anti-HA antibodies. Uncropped images of all Western blots are shown in Supplementary Figures 9-12

sumoylation of either PML/RARA $\mathrm{F} 52 / 54 \mathrm{E}_{\mathrm{E}}$ or PML/RARA $\mathrm{L}_{\mathrm{L} 3 \mathrm{E}}$ in $\mathrm{Pml}^{-1-}$ MEFs (Fig. 6b,c). PML/RARA $\mathrm{F52/54 \textrm {E }}$ or PML/RARA $\mathrm{L}_{\mathrm{L} 3 \mathrm{E}}$ efficiently transformed primary progenitors ex vivo-as assessed by increased clonogenic activity in semi-solid cultures. Yet, the mutants failed to undergo any arsenic-triggered NB re-assembly and terminal differentiation (Fig. 6d and Supplementary Figure $8 \mathrm{a}, \mathrm{b})$. Collectively, these data establish the requirement of RING tetramerization in APL development in vivo and arsenic response ex vivo.

\section{Discussion}

PML RING, B boxes and coiled coil domains cooperate to assemble the insoluble NB scaffolds. We discovered that PML RING assembles into tetrameric torus whose formation requires highly evolutionarily conserved PML-specific residues located on the two contact points (Fig. 1b). Their mutations, which do not alter RING folding, abolish PML NB assembly, demonstrating the role of tetramers in higher order of macromolecular assembly (Figs. 2-4). These are likely stabilized by the C-terminal helix bundles, common to the RINGs of other TRIMs. Within the tetramer, L73 is absolutely essential, while the four $\mathrm{SD} 1_{\mathrm{FQF}} /$ SD2 contacts quantitatively modulate NB-formation and sumoylation, most likely through tetramer stability (Fig. 5 and Supplementary Figure 7a). Conformation of the extended loop containing $\mathrm{SD} 1_{\mathrm{FQF}}$ could be regulated by phosphorylation of multiple serine residues in the uncommonly long PML Nterminus $^{34}$ (Fig. 1b and Supplementary Figure 3). PML RING tetramers constitute essential building blocks within larger macro-molecular scaffolds, directly or indirectly ensuring efficient UBC9 recruitment. PML sumoylation does not contribute to NBbiogenesis². RING K65 sumoylation is unessential for NBbiogenesis (Supplementary Figure 6d), but could stabilize UBC9 binding $^{2}$ and favor its activity towards other PML sites, notably K160. Arsenic, by targeting the B2 box $^{35}$, accelerates PML NB formation and subsequent sumoylation, a process abolished when the torus interfaces are disrupted (Figs. 5,6 and Supplementary Figure 6,7). The fact that wt PML not only rescues $\mathrm{PML}_{\mathrm{F} 52 / 54 \mathrm{E}} \mathrm{NB}$ formation but also its sumoylation supports the idea that PML sumoylation occurs in trans within mixed tetramer. Other TRIM proteins are endowed with RING-dependent E3-ubiquitin-ligase activities, dependent on their dimerization ${ }^{12-14}$. We propose that higher order PML RING assembly has endowed PML with the ability to yield NBs, recruit UBC9 and promote PML sumoylation, thus contributing to the functional diversification of the biochemical activity of TRIM family members.

\section{Methods}

Protein expression and purification. The pET32a vector encoding the PML $\mathrm{RING}_{49-104}$ domain (amino acids $\left.49-104\right)^{36}$ and a longer RING 1-119 (amino acids 1-119) were transformed into Escherichia coli BL21 (DE3) cells (Sangon) for protein production. The design of primer used in this study is shown in Supplementary Table 1 and 2 . The recombinant protein containing a $\mathrm{N}$-terminal cleavable (His) $_{6}$ tag was induced with $200 \mu \mathrm{M}$ IPTG (Sangon) and $20 \mu \mathrm{M} \mathrm{ZnCl}_{2}$ (Sangon) when the reading of $\mathrm{OD}_{600}$ reaches 0.8 . The cells were grown at $22^{\circ} \mathrm{C}$ for $14 \mathrm{~h}$ before harvest by centrifugation $(4700 \mathrm{~g}, 20 \mathrm{~min})$.

The bacterial cells were resuspended in buffer containing ( $20 \mathrm{mM}$ Tris, $100 \mathrm{mM}$ $\mathrm{NaCl}, \mathrm{pH} 8.0)$ and lysed using a cell cracker (JNBIO) applying $\left(20 \mathrm{~kg} \mathrm{~cm}^{-2}\right)$ pressure. Cell debris was removed by centrifugation and clean lysate was loaded onto a pre-equilibrated nickel sepharose column (His Trap HP, GE Healthcare). The column was washed with buffer containing $(20 \mathrm{mM}$ Tris, $20 \mathrm{mM}$ Imidazole, $500 \mathrm{mM} \mathrm{NaCl}, \mathrm{pH}$ 8.0) and PML RING was eluted with buffer containing (20 mM Tris, $150 \mathrm{mM}$ Imidazole, $100 \mathrm{mM} \mathrm{NaCl}, \mathrm{pH}$ 8.0). The TRX-His tag was removed by digestion with Thrombin enzyme at room temperature overnight after the eluate was dialyzed against Thrombin digestion buffer (Sigma). The cleaved TRX-His tag and uncleaved PML RING were removed by recycle over a pre-equilibrated nickel column. PML RING was purified further with an anion exchange sepharose column (Q HP, GE Healthcare) and a hydrophobic interaction sepharose column (Phenyl HP, GE Healthcare). The correct mass of the protein was confirmed by MS analysis, and the purity was checked by SDS-PAGE.

Crystallization and data collection. PML RING crystals were grown in 48-well plates using the vapor diffusion technique. Then $0.5-\mu \mathrm{l}$ PML RING $\left(26 \mathrm{mg} \mathrm{ml}^{-1}\right)$ was mixed with $0.5 \mu \mathrm{l}$ reservoir solution ( $500 \mathrm{mM}$ Ammonium Sulfate, $1 \mathrm{M}$ Lithium Sulfate and $100 \mathrm{mM}$ Sodium Citrate), and the plates were incubated at $4{ }^{\circ} \mathrm{C}$ for $\sim 2$ weeks. The crystals were stabilized in a 50:50 mixture of paraffin and paratone-N (Hampton Research), and then were flash-cooled in liquid nitrogen. Diffraction data were collected in Beamline station BL17U at Shanghai Synchrotron Radiation Facility (SSRF, Shanghai, China).

Phasing and structure refinement. The diffraction data were recorded at the wavelength of $\mathrm{Zn}$ anomalous dispersion peak (1.2824 $\AA$ ) and subsequently processed, integrated and scaled using MOSFLM/SCALA ${ }^{25}$. The statistics of the data collection are shown in Supplementary Table 1. Single wavelength dispersion (SAD) method implemented in CRANK2 ${ }^{25}$ was used to phase PML RING. Eight $\mathrm{Zn}^{2+}$ positions were determined by CRUNCH2 $2^{25}$ using data between 20 and $1.6 \AA$ An interpretable map was obtained by solvent flattening using program SOLO$\mathrm{MON}^{25}$. The autotracing program ARP/wARP was then used to produce a $\sigma_{\mathrm{A}^{-}}$ weighted $2 \mathrm{Fo}-\mathrm{Fc}$ map for further manual model building. REFMAC5 ${ }^{37}$ was used for structural refinement. Intermittent manual building implemented in COOT $^{25}$ was used to correct and improve the initial models produced by ARP/wARP ${ }^{38}$. The B-factors were refined with TLS corrections (4 TLS group, 84 parameters) ${ }^{37}$. The final model of PML RING tetramer contains 187 residues and 224 water molecules. Ramachandran statistics of PML RING calculated by PROCHECK ${ }^{39}$ indicate that $99.4 \%$ of the atoms are in the most favored region, and $0.6 \%$ are in the allowed regions. The detailed structure refinement statistics are reported in Table 1.

Analytical ultracentrifugation and gel filtration analysis. Sedimentation experiments were conducted using a Beckman XL-I Optima analytical ultracentrifuge equipped with absorbance optics. Sedimentation studies were carried out at $200,000 \mathrm{~g}$, at $25^{\circ} \mathrm{C}$ overnight. Three-channel with quartz windows were filled with $400 \mu \mathrm{l}$ of sample $(20 \mathrm{mM}$ Tris, $100 \mathrm{mM} \mathrm{NaCl}, \mathrm{pH} 8.0$, with/without $1 \mathrm{mM}$ DTT) at the concentration of $1 \mathrm{mg} \mathrm{ml}^{-1}$. To investigate how protein concentration might influence PML RING tetramerization, the wild type protein at the higher 
a
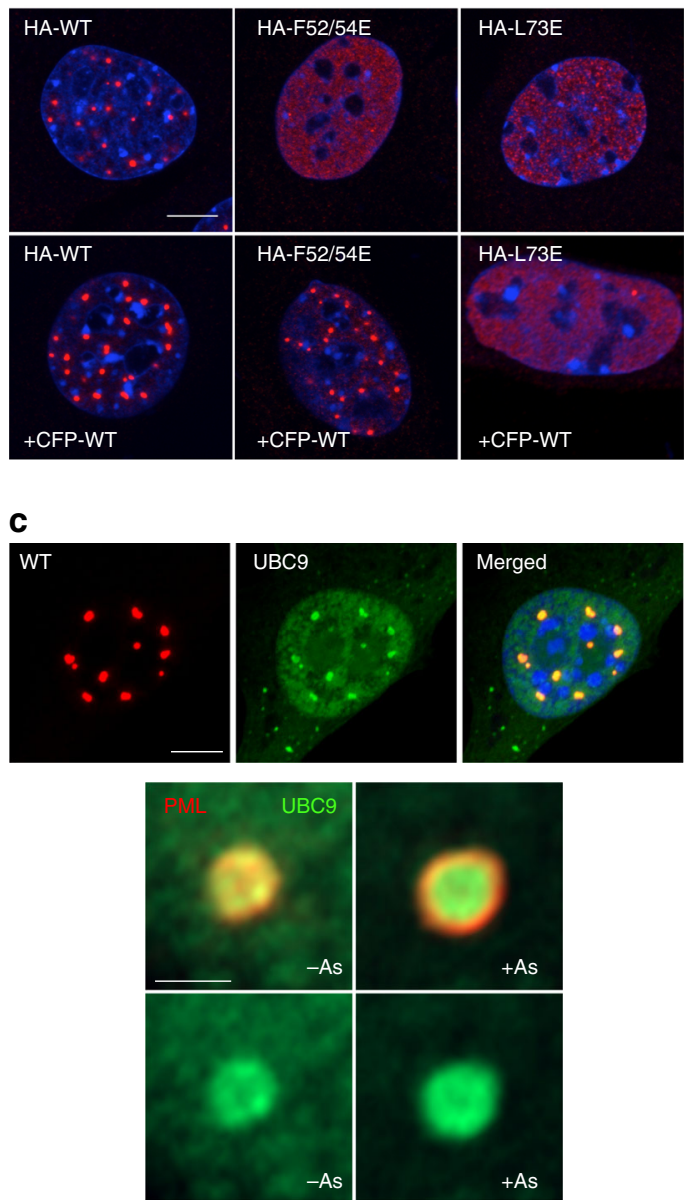

b

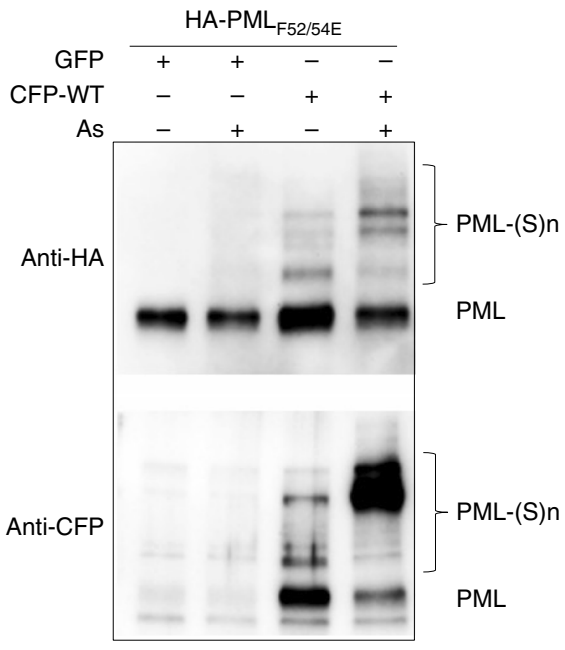

d

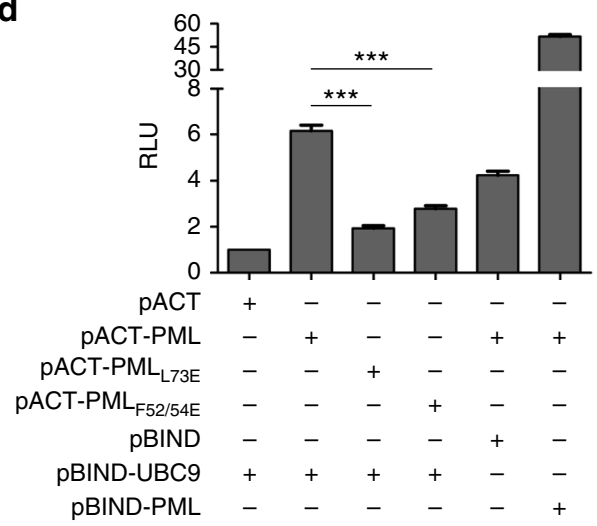

Fig. 5 PML tetramerization controls NB formation and PML sumoylation. a Immortalized Pm/ ${ }^{-/-}$MEFs were transduced with MSCV virus expressing PML or its mutants and subsequently transduced or not with CFP-PML. PML NBs were monitored by immuno-fluorescence using anti-HA antibody (red). DAPI is in blue. Scale bar is $5 \mu \mathrm{m}$. $\mathbf{b}$ Immortalized Pml-/- MEFs obtained in a were treated with $\mathrm{As}_{2} \mathrm{O}_{3}\left(10^{-6} \mathrm{M}, 1 \mathrm{~h}\right)$ and extracts were analyzed by Western blot using anti-HA or anti-CFP. PML and its sumoylated forms are indicated. c Co-localization of stably expressed HA-PML (red) and UBC9-GFP (green) basal condition (left) or upon $10^{-6} \mathrm{M} \mathrm{As}_{2} \mathrm{O}_{3}$ for $\mathrm{Th}$ (right). DAPI is in blue. Middle and bottom: Visualization of PML and UBC9 localization with/without arsenic. Scale bars are $5 \mu \mathrm{m}$ (top) and $0.5 \mu \mathrm{m}$ (middle and bottom). d Mammalian two-hybrid: relative luciferase activities (RLU) were used to estimate the interaction between UBC9 and PML/mutants. Statistical significance is indicated. All experiments have been done at least with three independent replicates. Values are means \pm S.E. ${ }^{\star \star \star} p<0.001$ are used to show statistically significant between recombinant derivatives. $\mathrm{pACT}-\mathrm{PML}$ and $\mathrm{pBIND}-\mathrm{PML}$ interaction is shown as a positive control

concentration $\left(5 \mathrm{mg} \mathrm{ml}^{-1}\right)$ was also tested. Absorbance profiles were acquired at a wavelength of $335 \mathrm{~nm}$, chosen according to the protein concentration. Data analysis was carried out using SEDFIT ${ }^{40}$, which employs the continuous $\mathrm{c}(\mathrm{s})$ conformational change model based on the Lamm equation, to determine the sedimentation coefficient distribution.

In order to check RING tetramerization in the context of full-length PML protein, gel filtration analysis was used. Recombinant proteins including PML $\triangle B C$ (i.e., the deletion of residues, 120-360) and mutants were obtained by in vitro translation (Promega) using pcDNA3.1(-) vector with T7 promoter according to manufacturer's standard protocol. The sample was then subjected to gel filtration analysis using Superose 6 and Superose 12 columns (GE) at a flow rate of $0.4 \mathrm{ml}$ $\mathrm{min}^{-1}$. Each fraction was monitored by Western blot using antibody against PML (Abcam).

Cell culture and treatments. For expression in MEFs or progenitors, MSCV retroviral constructs were used, HA or CFP tags were in frame with 5' PML coding sequence. Mutations were generated with the QuikChange II site-directed mutagenesis kit (QIAGEN) on MSCV-HA-His 6 -PML or pSG5-HA-His ${ }_{6}-\mathrm{PML}$ and subcloned in MSCV-HA-His ${ }_{6}$-PML (named HA-PML) or in MSCV-HA-His ${ }_{6}$ PML/RARA (HA-PML/RARA). Oligonucleotides used in all the constructs arelisted in Supplementary Table 2.

Immortalized $\mathrm{Pml}^{-/-}$MEF cells, obtained previously by large T expression in primary $\mathrm{MEFs}^{35}$, were transduced with MSCV virus expressing $\mathrm{HA}^{-\mathrm{His}_{6}}$-tagged
WT or mutants. For rescue experiments, MEFs stably expressing HA-PMLs were transduced with MSCV-CFP-WT PML or MSCV-GFP. Immortalized $\mathrm{Pml}^{-/-} \mathrm{MEF}$ stably expressing HA-WT PML (or indicated mutants) were transduced with MSCV virus expressing UBC9-GFP as described in the figures. MEFs were treated with $10^{-6} \mathrm{M} \mathrm{As}_{2} \mathrm{O}_{3}$ (Fluka) for $1 \mathrm{~h}$ before protein extraction and Western blot analysis using anti-HA, anti-CFP, anti-GFP, and anti-Lamin antibodies.

Mouse haematopoietic progenitors (i.e., lineage-depleted bone marrow from 5fluorouracil-treated C57BL/6 mice) were transduced with MSCV-HA-His 6 -PML/ RARA or its mutants, cultured in RPMI medium supplemented with IL-3, IL-6 and stem cell factor and treated with $10^{-6} \mathrm{M} \mathrm{As}_{2} \mathrm{O}_{3}$ for $1 \mathrm{~h}$. For FACS and MGG experiments, transduced cells were re-plated in methylcellulose at a density of 10,000 cells/dish in the presence or absence of $10^{-7} \mathrm{M} \mathrm{As}_{2} \mathrm{O}_{3}$. Cells were analyzed after 7 days using FACS according to manufacturer's guidelines and MGG staining.

Immunoflorescence image acquision and Western blot. Cells were fixed with $4 \%$ paraformaldehyde. Immunofluorescence assays were performed and analysed by confocal microscopy using the antibodies described below. The slides were examined with a Leica TCS SP8 or Zeiss LSM870 confocal fluorescent microscope. Protein extracts were prepared by lysing cells directly in Laemmli buffer. SUMO conjugates and PML proteins were separated on $4-12 \%$ gradient SDS-PAGE (Biorad). Homemade chicken polyclonal anti-human PML was previously described $^{41}$. Mouse monoclonal anti-HA was from Covance, anti-GFP from Roche, rabbit polyclonal anti-CFP and goat polyclonal anti-lamin B antibodies from 

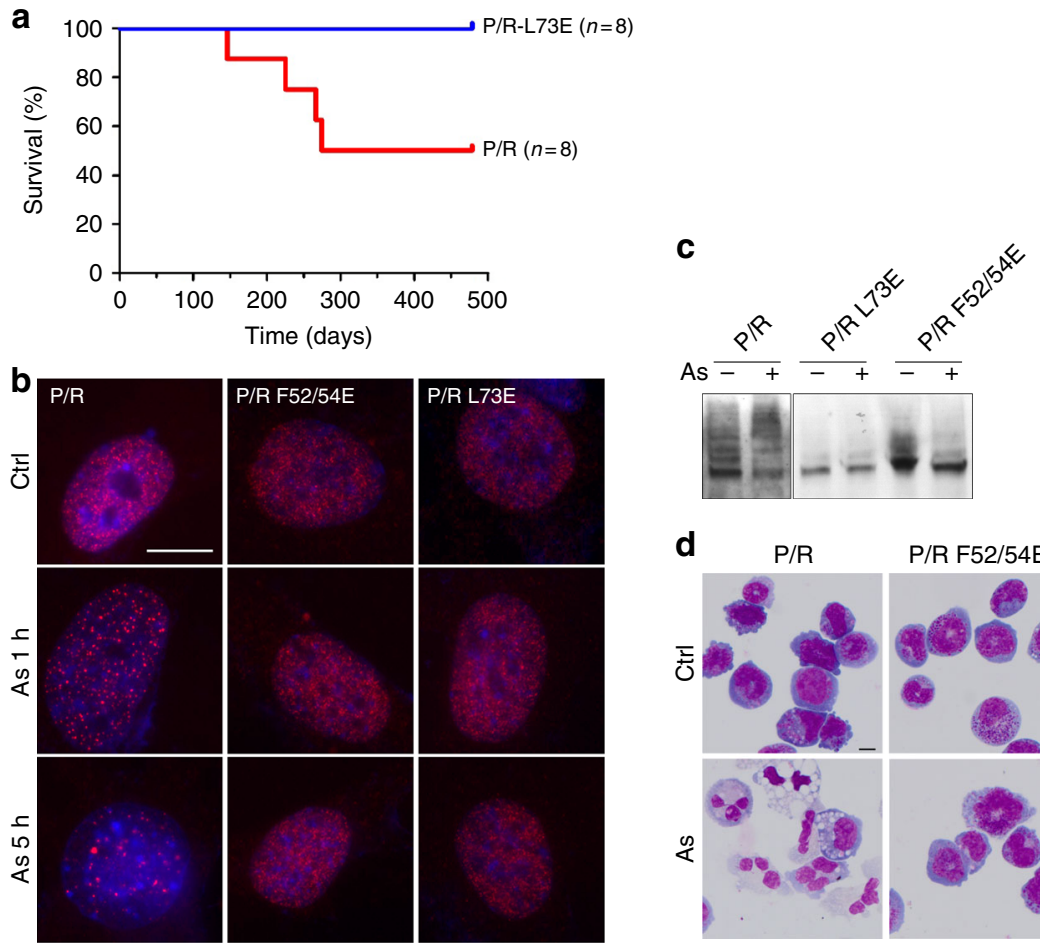

d

$\mathrm{P} / \mathrm{R}$

P/R F52/54E

P/R L73E

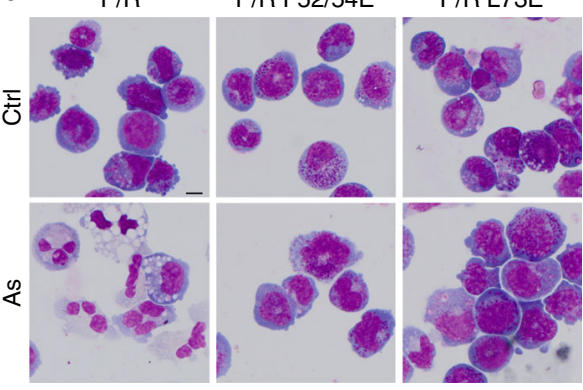

e

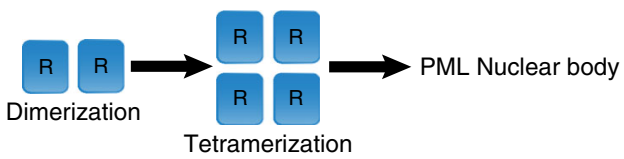

Sumoylation

Fig. 6 PML RING tetramerization is important for APL development and arsenic targeting of PML/RARA-transformed cells. a Survival data of MRP8-PML/

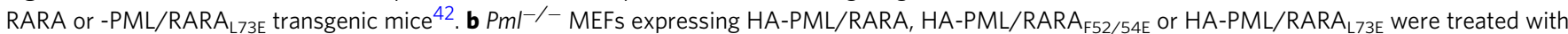
$\mathrm{As}_{2} \mathrm{O}_{3}\left(10^{-6} \mathrm{M}\right)$ prior to immunofluorescence analysis. Scale bar is $5 \mu \mathrm{m}$. c Sumoylation of HA-PML/RARA after $1 \mathrm{~h}$ of $\mathrm{As}_{2} \mathrm{O}_{3}$ exposure was monitored by Western blot using anti-RARA antibody ${ }^{41}$. d MGG staining of mouse hematopoietic progenitors transformed by PML/RARA and the indicated mutants after arsenic treatment $\left(10^{-7} \mathrm{M}, 7\right.$ days). e Proposed model for NB assembly and PML sumoylation integrating the PML RING tetramer formation

Sigma-Aldrich. Anti-mouse cKit (CD117) and anti-mouse Mac1 antibodies were from BD pharmingen. Alexa 488- or 594-labeled secondary antibodies and HRPconjugated secondary antibodies from Jackson Laboratories.

For statistics, PML NBs were counted from at least 50 randomly chosen cells. The ratio between sumoylated and unmodified PML was calculated from Vilber Lourmat Fusion camera software. All experiments have been done at least with three independent replicates.

FRAP analysis. Fluorescence recovery after photobleaching (FRAP) was performed on Zeiss LSM510 confocal microscope equipped with a heated chamber.

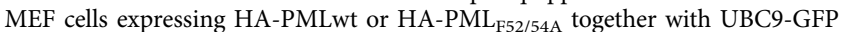
were seeded in glass-bottomed dish in HEPES-buffered DMEM media with 10\% FBS and the dish was mounted on the stage in the confocal chamber pre-heated to $37^{\circ} \mathrm{C}$. The cells were observed under a $63 \times$ oil lens. The "region of interest (ROI)" containing only one PML NB was selected, and UBC9-GFP was bleached with a $488 \mathrm{~nm}$ argon laser at 100\% intensity for 6 times. The means of relative intensity of UBC9-GFP fluorescence during fluorescent recovery were quantified from three independent experiments.

Transgenic mice. PML/RARA and PML/RARA $A_{\mathrm{L} 73 \mathrm{E}}$ were expressed using human MRP8 promoter ${ }^{42}$. Mice were housed in specific pathogen-free conditions and all animal experiments were approved by the Animal Care and Use Committee at Experimental Animal Center in Shanghai Jiao Tong University School of Medicine. To define whether the mice have developed APL, hematological disorders such as splenomegaly, MGG staining and flow cytometry analysis of c-Kit, GR1 and Mac-1 in spleen and bone marrow cells were performed.

Circular dichroism. Samples for CD were prepared by desalting the bacterial expressed recombinant proteins and diluting them to $0.2 \mathrm{mg} \mathrm{ml}^{-1}$ using $10 \mathrm{mM}$ CHES buffer (pH 9.0). Far UV CD spectra was recorded from $185 \mathrm{~nm}$ to $260 \mathrm{~nm}$ at $20^{\circ} \mathrm{C}$ with a time constant of $1 \mathrm{~s}$ using a Chirascan spectrometer (Applied Photophysics Ltd). The spectra were the average of not less than three scans and presented as mean residue molar ellipticity $[\theta]\left(\right.$ deg.cm $\left.{ }^{2} \mathrm{dmol}^{-1}\right)$. Secondary structure content reported in Supplementary Figure $2 \mathrm{~b}$ was estimated using Circular dichroism (CD) deconvolution program CDNN.

SEC-MALS analysis. The purified PML RING ${ }_{1-119}$ and mutants were subjected to gel filtration analysis (S100 column, GE Healthcare). The elution peaks, as monitored by UV absorption at $280 \mathrm{~nm}$, were pooled separately and chosen for size exclusion chromatography-multi-angle light scattering (SEC-MALS) characterization, respectively. In brief, the purified protein samples were concentrated and analyzed using a WTC-015S5 sized exclusion column (Wyatt Technology) which was connected to a 1260 infinity liquid chromatography system (Agilent Technology) equipped with inline DAWN HELEOS-II MALS and Optilab rEX differential refractive index detectors (Wyatt Technology). For each sample, a $40 \mu \mathrm{l}$ injection volume and $0.5 \mathrm{ml} \mathrm{min}^{-1}$ flow rate were applied. Data were recorded and processed using ASTRA VI software (Wyatt Technology).

Small angle X-ray scattering. The recombinant PML RING $49-104$ was purified and concentrated to 1,3 and $5 \mathrm{mg} \mathrm{ml}^{-1}$ in $20 \mathrm{mM}$ Tris, $\mathrm{pH} 8.0,100 \mathrm{mM} \mathrm{NaCl}$, with/without $1 \mathrm{mM}$ DTT, respectively. The X-ray scattering experiment was carried out at Beamline station BL19U2 (National Facility for Protein Science Shanghai, NCPSS, China) and scattered X-ray intensities were collected by using a Pilatus $1 \mathrm{M}$ detector (DECTRIS Ltd). The measurements were carried out with $1 \mathrm{~s}$ exposure time and repeat for 20 times to avoid possible sample radiation damage. The collected data were processed with ATSAS software package ${ }^{43}$. The details of SAXS data collection and processing are shown in Supplementary Table 3. The guinier region of experimental group are linear, indicating the measured sample is homogeneous in solution. Crystal data fitting was done using the OLIGOMER algorism implemented in CRYSOL. In the analysis of crystal fitting $(0.01 \leq q \leq 0.25$ $\AA^{-1}$ ), the crystallographic tetramer, crystallographic dimers and crystallographic and NMR monomers were used to fit the merged experimental SAXS data derived from three protein concentrations, leading to the determination of each fraction of monomer/dimer/tetramer in the solutions. 


\section{Table 1 Data collection and structure refinement statistics of PML-RING}

\section{Data collection}

Space group

Unit cell dimension $(\AA)$

a

b

Molecule per ASU

Derivative

Source/Station ${ }^{\text {a }}$

Wavelength $(\AA)$

Resolution range $(\AA)$

Observations $(I / \sigma(I)>0)$

Unique reflections $(I / \sigma(I)>0)$

High resolution shell $(\AA)$

$R_{\text {sym }}(\%)^{b, c}$

Completeness ${ }^{\mathrm{c}}(\%)$

Redundancy ${ }^{\mathrm{C}}$

$\mathrm{CC}_{1 / 2}$

Structure refinement

Resolution range $(\AA)$

$R$-factor (\%)

$R$-factor (high resolution shell) ${ }^{\mathrm{d}}$

$R_{\text {free }}(\%)^{\mathrm{e}}$

$R_{\text {free }}$ (high resolution shell)

Total number of non-hydrogen atoms

Protein atoms

Water molecules

Zn ions

R.m.s. deviations

Bond length $(\AA)$

Bond angle $\left({ }^{\circ}\right)$

Main chain $B$-factors $\left(\AA^{2}\right)$

Side chain $B$-factors $\left(\AA^{2}\right)$

Wilson $B$-factor $\left(\AA^{2}\right)$

Average $B$-factor $\left(\AA^{2}\right)$

Protein atoms

Solvent atoms

$\mathrm{Zn}$ ions

$\mathrm{P} 2{ }_{1} 2_{1} 2_{1}$

38.5

84.7

86.1

4

Native

BL17U

1.2824, $\mathrm{Zn}_{\text {peak }}$

$60.4-1.60$

467248

37754

$1.69-1.60$

$14.9(133.6)<I / \sigma(I)>c: 8.0(1.3)$

$99.4(99.9)$

$2.4(11.5)$

$0.996(0.848)$

$60.4-1.60$

20.3

35.0

21.7

36.6

1403

224

8

0.005

0.954

1.799

5.871

22.1

43.8

49.5

38.3

Ramachandran statistics (\%)

Most favored region

Allowed regions

99.4

0.6

Beamline designations refer to the Shanghai Synchrotron Radiation Facility, Shanghai, P. R. of China

${ }^{\mathrm{b}} R_{\text {sym }}=\Sigma(I-\langle|>)^{2} /\left.\Sigma\right|^{2}$

overall, high resolution shell in parenthese

dhigh resolution shell: $1.640-1.600 \AA$

${ }^{e} R_{\text {free }}$ calculated using $5 \%$ of total reflections omitted from refinement

R.m.s. deviations report root mean square deviations from ideal bond lengths/angles and of $B$ factors between bonded atoms ${ }^{44}$

Mammalian two-hybrid assay. Mammalian two-hybrid assay was performed using the CheckMate ${ }^{\mathrm{TM}}$ Mammalian Two-Hybrid System (Promega) in $293 \mathrm{~T}$ or CHO cells. The cDNA of UBC9 and full length WT PML or mutants were inserted into pBIND and pACT vectors, respectively. 293T cells were then transfected with plasmids pG5/luc, pBIND-UBC9 and pACT-PML/mutant (mixed at a molar ratio of 1:1:1) using liposome Lipo2000 transfection method (Life Technology). In the complementary set of experiments, UBC9 was cloned into pACT and the extended RING domain into pBIND. The resulting plasmids were transfected in $\mathrm{CHO}$ cells using Effecten (Quiagen). Twenty-four hours after transfection, luciferase activity was determined using the Dual-Luciferase Reporter Assay System (Promega).

Limited proteolysis. $10 \mu \mathrm{g}$ purified PML RING ${ }_{1-119}$ was subjected to chymotrypsin (Sigma) digestion at $25^{\circ} \mathrm{C}$ for $20 \mathrm{~min}$. The reaction mixture contained 10 $\mu \mathrm{g} \mathrm{RING}_{1-119}$, a series dilutions from $10 \mu \mathrm{g}$ chymotrypsin, $100 \mathrm{mM}$ Tris ( $\mathrm{pH} 8.0$ ), $10 \mathrm{mM} \mathrm{CaCl}_{2}$ and $1 \mathrm{mM}$ DTT. The reaction was terminated by boiling with SDS loading buffer. The sample was analyzed by SDS-PAGE followed by silver staining.

Mass spectrometry analysis was conducted with 5800 MALDI-TOF/TOF (AB Sciex). The limited digestion product was subjected to the PVDF (GE Healthcare) membrane transferring followed by Ponceau S staining to indicate the target protein band. Cropped PVDF membrane containing the target protein was placed into the reactor of the PPSQ-33A (SHIMADZU) automatic protein sequencer, followed by a standard analysis procedure.
Data availability. Coordinates and structure factors have been deposited in the Protein Data Bank under accession code 5YUF. Other data are available from the corresponding authors upon request.

Received: 22 November 2016 Accepted: 20 February 2018

Published online: 29 March 2018

\section{References}

1. Lallemand-Breitenbach, V. \& de The, H. PML nuclear bodies. Cold Spring Harb. Perspect. Biol. 2, a000661 (2010).

2. Sahin, U. et al. Oxidative stress-induced assembly of PML nuclear bodies controls sumoylation of partner proteins. J. Cell. Biol. 204, 931-945 (2014).

3. Reuter, N., Schilling, E.M., Scherer, M., Muller, R., Stamminger, T. The ND10 component promyelocytic leukemia protein acts as an E3 ligase for SUMOylation of the major immediate early protein IE1 of human cytomegalovirus. J. Virol. 91, 2335-16 (2017).

4. Pearson, M. et al. PML regulates p53 acetylation and premature senescence induced by oncogenic Ras. Nature 406, 207-210 (2000).

5. de The, H., Le Bras, M. \& Lallemand-Breitenbach, V. The cell biology of disease: acute promyelocytic leukemia, arsenic, and PML bodies. J. Cell. Biol. 198, 11-21 (2012)

6. Niwa-Kawakita, M. et al. PML is a ROS sensor activating p53 upon oxidative stress. J. Exp. Med. 214, 3197-3206 (2017)

7. de The, H. \& Chen, Z. Acute promyelocytic leukaemia: novel insights into the mechanisms of cure. Nat. Rev. Cancer 10, 775-783 (2010).

8. Ablain, J. et al. Activation of a promyelocytic leukemia-tumor protein 53 axis underlies acute promyelocytic leukemia cure. Nat. Med. 20, 167-174 (2014).

9. de The, H., Pandolfi, P. P. \& Chen, Z. Acute promyelocytic leukemia: a paradigm for oncoprotein-targeted cure. Cancer Cell 32, 552-560 (2017).

10. Reymond, A. et al. The tripartite motif family identifies cell compartments. EMBO J. 20, 2140-2151 (2001).

11. Meroni, G. \& Diez-Roux, G. TRIM/RBCC, a novel class of 'single protein RING finger' E3 ubiquitin ligases. Bioessays 27, 1147-1157 (2005)

12. Hatakeyama, S. TRIM proteins and cancer. Nat. Rev. Cancer 11, 792-804 (2011).

13. Streich, F. C. Jr, Ronchi, V. P., Connick, J. P. \& Haas, A. L. Tripartite motif ligases catalyze polyubiquitin chain formation through a cooperative allosteric mechanism. J. Biol. Chem. 288, 8209-8221 (2013).

14. Yudina, Z. et al. RING dimerization links higher-order assembly of TRIM5alpha to synthesis of K63-linked polyubiquitin. Cell Rep. 12, 788-797 (2015).

15. Duprez, E. et al. SUMO-1 modification of the acute promyelocytic leukaemia protein PML: implications for nuclear localisation. J. Cell Sci. 112, 381-393 (1999).

16. Borden, K. L. et al. The solution structure of the RING finger domain from the acute promyelocytic leukaemia proto-oncoprotein PML. EMBO J. 14, 1532-1541 (1995)

17. Borden, K. L. et al. In vivo and in vitro characterization of the B1 and B2 zincbinding domains from the acute promyelocytic leukemia protooncoprotein PML. Proc. Natl. Acad. Sci. USA 93, 1601-1606 (1996).

18. Boddy, M. N., Duprez, E., Borden, K. \& Freemont, P. S. Surface residue mutations of the pml ring finger domain alter the formation of nuclear matrix-associated pml bodies. J. Cell Sci. 110, 2197-2205 (1997).

19. Muller, S., Matunis, M. J. \& Dejean, A. Conjugation with the ubiquitin-related modifier SUMO-1 regulates the partitioning of PML within the nucleus. EMBO J. 17, 61-70 (1998).

20. Shen, T. H., Lin, H. K., Scaglioni, P. P., Yung, T. M. \& Pandolfi, P. P. The mechanisms of PML-nuclear body formation. Mol. Cell 24, 331-339 (2006)

21. Lallemand-Breitenbach, V. et al. Role of promyelocytic leukemia (PML) sumolation in nuclear body formation, 11S proteasome recruitment, and As2O3-induced PML or PML/retinoic acid receptor alpha degradation. J. Exp. Med. 193, 1361-1371 (2001).

22. Cuchet-Lourenco, D. et al. SUMO pathway dependent recruitment of cellular repressors to herpes simplex virus type 1 genomes. PLoS Pathog. 7, e1002123 (2011).

23. Banani, S. F. et al. Compositional control of phase-separated cellular bodies Cell 166, 651-663 (2016).

24. Huang, S. Y. et al. The RING domain of human promyelocytic leukemia protein (PML). J. Biomol. Nmr. 61, 173-180 (2015).

25. Collaborative Computational Project, Number 4. The CCP4 suite: programs for protein crystallography. Acta Crystallogr. D Biol. Crystallogr. 50, 760-763 (1994).

26. Dawidziak, D. M., Sanchez, J. G., Wagner, J. M., Ganser-Pornillos, B. K. \& Pornillos, O. Structure and catalytic activation of the TRIM23 RING E3 ubiquitin ligase. Proteins 85, 1957-1961 (2017). 
27. Keown, J. R., Yang, J. X., Douglas, J. \& Goldstone, D. C. Characterisation of assembly and ubiquitylation by the RBCC motif of Trim5alpha. Sci. Rep. 6, 26837 (2016)

28. Sanchez, J. G. et al. Mechanism of TRIM25 catalytic activation in the antiviral RIG-I pathway. Cell Rep. 16, 1315-1325 (2016).

29. Koliopoulos, M. G., Esposito, D., Christodoulou, E., Taylor, I. A. \& Rittinger, K. Functional role of TRIM E3 ligase oligomerization and regulation of catalytic activity. Embo. J. 35, 1204-1218 (2016).

30. Zhu, J. et al. A sumoylation site in PML/RARA is essential for leukemic transformation. Cancer Cell. 7, 143-153 (2005).

31. Nasr, R. et al. Eradication of acute promyelocytic leukemia-initiating cells through PML-RARA degradation. Nat. Med. 14, 1333-1342 (2008).

32. Zhu, J. et al. Arsenic-induced PML targeting onto nuclear bodies: implications for the treatment of acute promyelocytic leukemia. Proc. Natl. Acad. Sci. USA 94, 3978-3983 (1997).

33. Chen, G. Q. et al. Use of arsenic trioxide (As2O3) in the treatment of acute promyelocytic leukemia (APL): I. As2O3 exerts dose-dependent dual effects on APL cells. Blood 89, 3345-3353 (1997).

34. Hayakawa, F. \& Privalsky, M. L. Phosphorylation of PML by mitogenactivated protein kinases plays a key role in arsenic trioxide-mediated apoptosis. Cancer Cell 5, 389-401 (2004).

35. Jeanne, M. et al. PML/RARA oxidation and arsenic binding initiate the antileukemia response of As2O3. Cancer Cell 18, 88-98 (2010).

36. Zhang, X. W. et al. Arsenic trioxide controls the fate of the PML-RARalpha oncoprotein by directly binding PML. Science 328, 240-243 (2010).

37. Winn, M. D., Isupov, M. N. \& Murshudov, G. N. Use of TLS parameters to model anisotropic displacements in macromolecular refinement. Acta Crystallogr. D Biol. Crystallogr. 57, 122-133 (2001).

38. Perrakis, A., Morris, R. \& Lamzin, V. S. Automated protein model building combined with iterative structure refinement. Nat. Struct. Biol. 6, 458-463 (1999).

39. Laskowski, R. A., MacArthur, M. W., Moss, D. S. \& Thornton, J. M. PROCHECK: a program to check the stereochemical quality of protein structures. Jnl Appl. Crystallogr. 26, 283-291 (1993).

40. Schuck, P., Perugini, M. A., Gonzales, N. R., Howlett, G. J. \& Schubert, D. Size-distribution analysis of proteins by analytical ultracentrifugation: strategies and application to model systems. Biophys. J. 82, 1096-1111 (2002).

41. Lallemand-Breitenbach, V. et al. Arsenic degrades PML or PML-RARalpha through a SUMO-triggered RNF4/ubiquitin-mediated pathway. Nat. Cell Biol. 10, 547-555 (2008).

42. Brown, D. et al. A PML RAR alpha transgene initiates murine acute promyelocytic leukemia. Proc. Natl. Acad. Sci. USA 94, 2551-2556 (1997).

43. Franke, D. et al. ATSAS 2.8: a comprehensive data analysis suite for smallangle scattering from macromolecular solutions. J. Appl. Crystallogr. 50, 1212-1225 (2017).

44. Engh, R. A. \& Huber, R. Accurate bond and angle parameters for x-ray protein structure refinement. Acta Crystallogr. A47, 392-400 (1991).

\section{Acknowledgements}

This work was supported by research grants 81770142, 81370620, 81570120, 31070645 from National Scientific Foundation of China (to GM), "The Program for Professor of Special Appointment (Eastern Scholar) at Shanghai Institute of Higher Learning" (to GM), a research grant 20152504 from "Shanghai Municipal Education CommissionGaofeng Clinical Medicine Grant Support” (to GM), a research grant 11JC1407200 from
SMSTC (to GM), a research grant 12ZZ109 from SME (to GM), "Program for New Century Excellent Talents in University (NCET-10-9571)" (to GM). H.de Thé laboratory is supported by Ligue Contre le Cancer, INSERM, CNRS, Collège de France, Universite Paris-Diderot, Institut National du Cancer, Association pour la Recherche contre le Cancer (Griffuel Award to H. de Thé), the European Research Council (STEMAPL advanced grant to H. de Thé), the French National Research Agency (ANR) "Investissements d'Avenir" program (reference: ANR-11-PHUC-002) and SUMOPiv project. We thank the 'plateau technologique de l'IUH, notably Niclas Setterblad for help with the imaging facility. We thank the staffs from BL17U/BL18U1/BL19U1/BL19U2/BL01B beamline of National Center for Protein Sciences Shanghai (NCPSS) at SSRF, for assistance during data collection. We thank Dr. Xiaoxia Yu (Institute of Biophysics, Beijing, China) for help in sedimentation experiment. We thank Drs. Shaoyong Lu and Jian Zhang (Shanghai Jiao Tong University, School of Medicine) for assistance in MD analysis and colleagues, notably Morgane Le Bras and Cécile Esnault for careful reading of the manuscript.

\section{Author contributions}

Conceived and designed the experiments: V.L.B., Z.C., H.d.T. and G.M. Performed the experiments: P.W., S.B., H.W., V.L.B., T.Z., F.J., L.P., Y.L. Analyzed the data: P.W., S.B., H.W., V.L.B., T.Z., F.J., L.P., Y.L., S.J.C., Z.C., H.d.T., G.M. Preparation of the figures: P. W., S.B., H.W., V.L.B., T.Z., F.J., L.P., Y.L., S.J.C., Z.C., H.d.T., G.M. Wrote the paper: P. W., H.W., V.L.B., H.d.T., G.M.

\section{Additional information}

Supplementary Information accompanies this paper at https://doi.org/10.1038/s41467018-03498-0.

Competing interests: All authors declare no competing interests.

Reprints and permission information is available online at http://npg.nature.com/ reprintsandpermissions/

Publisher's note: Springer Nature remains neutral with regard to jurisdictional claims in published maps and institutional affiliations.

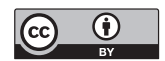

Open Access This article is licensed under a Creative Commons Attribution 4.0 International License, which permits use, sharing, adaptation, distribution and reproduction in any medium or format, as long as you give appropriate credit to the original author(s) and the source, provide a link to the Creative Commons license, and indicate if changes were made. The images or other third party material in this article are included in the article's Creative Commons license, unless indicated otherwise in a credit line to the material. If material is not included in the article's Creative Commons license and your intended use is not permitted by statutory regulation or exceeds the permitted use, you will need to obtain permission directly from the copyright holder. To view a copy of this license, visit http://creativecommons.org/ licenses/by/4.0/.

(C) The Author(s) 2018 